\title{
Intuitionistic Fuzzy Hx Ring
}

\author{
R. Muthuraj ${ }^{1}$, M. S. Muthuraman ${ }^{2}$ \\ ${ }^{I} P G \&$ Research Department of Mathematics, H.H.The Rajah's College, Pudukkottai-622 001, Tamilnadu, \\ India. \\ ${ }^{2}$ Department of Mathematics, PSNA College of Engineering and Technology, Dindigul-624 622, Tamilnadu , \\ India.
}

\begin{abstract}
In this paper, we define the notion of intuitionistic fuzzy sub HX ring of a HX ring and some of their related properties are investigated. We define the necessity and possibility operators of an intuitionistic fuzzy subset of an intuitionistic fuzzy $H X$ ring and discuss some of its properties. We introduce the concept of an image, pre-image of an intuitionistic fuzzy subset and discuss in detail a series of homomorphic and anti homomorphic properties of an intuitionistic fuzzy set are discussed.
\end{abstract}

Keywords: intuitionistic fuzzy set, fuzzy HX ring, intuitionistic fuzzy sub HX ring, homomorphism and anti homomorphism of an intuitionistic fuzzy HX ring, image and pre-image of an intuitionistic fuzzy set.

\section{Introduction}

In 1965, Zadeh [12] introduced the concept of fuzzy subset $\mu$ of a set $\mathrm{X}$ as a function from $\mathrm{X}$ into the closed unit interval $[0,1]$ and studied their properties. Fuzzy set theory is a useful tool to describe situations in which the data or imprecise or vague and it is applied to logic, set theory, group theory, ring theory, real analysis, measure theory etc. In 1967, Rosenfeld [11] defined the idea of fuzzy subgroups and gave some of its properties. Li Hong Xing [5] introduced the concept of HX group. In 1982 Wang-jin Liu[7] introduced the concept of fuzzy ring and fuzzy ideal. With the successful upgrade of algebraic structure of group many researchers considered the algebraic structure of some other algebraic systems in which ring was considered as first. In 1988, Professor Li Hong Xing [6] proposed the concept of HX ring and derived some of its properties, then Professor Zhong [2,3] gave the structures of HX ring on a class of ring. R.Muthuraj et.al[10] introduced the concept of fuzzy HX ring. In this paper we define a new algebraic structure of an intuitionistic fuzzy sub HX ring of a HX ring and investigate some related properties. We define the necessity and possibility operators of an intuitionistic fuzzy subset of an intuitionistic fuzzy HX ring and discuss some of its properties. Also we introduce the image and pre-image of an intuitionistic fuzzy set in an intuitionistic fuzzy HX ring and discuss some of its properties.

\section{Preliminaries}

In this section, we site the fundamental definitions that will be used in the sequel. Throughout this paper, $\mathrm{R}=(\mathrm{R},+, \cdot)$ is a Ring, e is the additive identity element of $\mathrm{R}$ and $\mathrm{xy}$, we mean $\mathrm{x} . \mathrm{y}$

\subsection{Definition [1]}

Let $\mathrm{R}$ be a ring. In $2^{\mathrm{R}}-\{\phi\}$, a non-empty set $\vartheta \subset 2^{\mathrm{R}}-\{\phi\}$ with two binary operation ' + ' and '.' is said to be a $\mathrm{HX}$ ring on $\mathrm{R}$ if $\vartheta$ is a ring with respect to the algebraic operation defined by

i. $\quad A+B=\{a+b / a \in A$ and $b \in B\}$, which its null element is denoted by $Q$, and the negative element of $\mathrm{A}$ is denoted by $-\mathrm{A}$.

ii. $\quad \mathrm{AB}=\{\mathrm{ab} / \mathrm{a} \in \mathrm{A}$ and $\mathrm{b} \in \mathrm{B}\}$,

iii. $\quad \mathrm{A}(\mathrm{B}+\mathrm{C})=\mathrm{AB}+\mathrm{AC}$ and $(\mathrm{B}+\mathrm{C}) \mathrm{A}=\mathrm{BA}+\mathrm{CA}$.

\subsection{Definition}

Let $\mathrm{R}$ be a ring. Let $\mu$ be a fuzzy ring defined on R. Let $\vartheta \subset 2^{\mathrm{R}}-\{\phi\}$ be a HX ring. A fuzzy subset $\lambda^{\mu}$ of $\vartheta$ is called a fuzzy HX ring on $\vartheta$ or a fuzzy ring induced by $\mu$ if the following conditions are satisfied. For all $\mathrm{A}, \mathrm{B} \in \vartheta$,
i. $\quad \lambda^{\mu}(A-B) \geq \min \left\{\lambda^{\mu}(A), \lambda^{\mu}(B)\right\}$,
ii. $\quad \lambda^{\mu}(\mathrm{AB}) \geq \min \left\{\lambda^{\mu}(\mathrm{A}), \lambda^{\mu}(\mathrm{B})\right\}$
where $\lambda^{\mu}(\mathrm{A})=\max \{\mu(\mathrm{x}) /$ for all $\mathrm{x} \in \mathrm{A} \subseteq \mathrm{R}\}$. 


\subsection{Definition}

\section{Properties of an intuitionistic fuzzy HX subring}

Let $\mathrm{R}$ be a ring. Let $\mu$ be a fuzzy ring on $\mathrm{R}$ and a nonempty set $\vartheta \subset 2^{\mathrm{R}}-\{\phi\}$ is a HX ring. An intuitionistic fuzzy subset $\psi=\left\langle\mathrm{A}, \lambda^{\mu}(\mathrm{A}), \lambda^{\gamma}(\mathrm{A})\right\rangle$ of a HX ring $\vartheta$ is said to be an intuitionistic fuzzy HX (IFHX) subring of $\vartheta$ if the following conditions are satisfied. For all $A, B \in \vartheta$,

(i) $\quad \lambda^{\mu}(\mathrm{A}-\mathrm{B}) \quad \geq \min \left\{\lambda^{\mu}(\mathrm{A}), \lambda^{\mu}(\mathrm{B})\right\}$,

(ii) $\quad \lambda^{\mu}(\mathrm{AB}) \quad \geq \min \left\{\lambda^{\mu}(\mathrm{A}), \lambda^{\mu}(\mathrm{B})\right\}$,

(iii) $\quad \lambda^{\gamma}(\mathrm{A}-\mathrm{B}) \quad \leq \max \left\{\lambda^{\gamma}(\mathrm{A}), \lambda^{\gamma}(\mathrm{B})\right\}$,

(iv) $\quad \lambda^{\gamma}(\mathrm{AB}) \leq \max \left\{\lambda^{\gamma}(\mathrm{A}), \lambda^{\gamma}(\mathrm{B})\right\}$

Where $\lambda^{\mu}(\mathrm{A})=\max \{\mu(\mathrm{x}) / \mathrm{x} \in \mathrm{A} \subseteq \mathrm{R}\} \quad, \lambda^{\gamma}(\mathrm{A})=\min \{\gamma(\mathrm{x}) / \mathrm{x} \in \mathrm{A} \subseteq \mathrm{R}\}$.

\subsection{Definition}

Let $R$ be a ring. Let $\mu$ be a fuzzy ring on $R$ and a nonempty set $\vartheta \subset 2^{R}-\{\phi\}$ is a HX ring. An intuitionistic fuzzy subset $\psi=\left\langle\mathrm{A}, \lambda^{\mu}(\mathrm{A}), \lambda^{\gamma}(\mathrm{A})\right\rangle$ of a $\mathrm{HX}$ ring $\vartheta$ is said to be an intuitionistic anti fuzzy HX (IAFHX) subring of $\vartheta$ if the following conditions are satisfied. For all $\mathrm{A}, \mathrm{B} \in \vartheta$,

(i) $\quad \lambda^{\mu}(\mathrm{A}-\mathrm{B}) \leq \max \left\{\lambda^{\mu}(\mathrm{A}), \lambda^{\mu}(\mathrm{B})\right\}$

(ii) $\quad \lambda^{\mu}(\mathrm{AB}) \leq \max \left\{\lambda^{\mu}(\mathrm{A}), \lambda^{\mu}(\mathrm{B})\right\}$,

(iii) $\quad \lambda^{\gamma}(\mathrm{A}-\mathrm{B}) \geq \min \left\{\lambda^{\gamma}(\mathrm{A}), \lambda^{\gamma}(\mathrm{B})\right\}$,

(iv) $\quad \lambda^{\gamma}(\mathrm{AB}) \quad \geq \min \left\{\lambda^{\gamma}(\mathrm{A}), \lambda^{\gamma}(\mathrm{B})\right\}$

Where $\lambda^{\mu}(\mathrm{A})=\min \{\mu(\mathrm{x}) / \mathrm{x} \in \mathrm{A} \subseteq \mathrm{R}\}, \lambda^{\gamma}(\mathrm{A})=\max \{\gamma(\mathrm{x}) / \mathrm{x} \in \mathrm{A} \subseteq \mathrm{R}\}$.

\subsection{Theorem}

If $\psi_{1}$ and $\psi_{2}$ be two intuitionistic fuzzy HX subrings of a HX ring $\vartheta$, then $\psi_{1} \cap \psi_{2}$ is also intuitionistic fuzzy HX subrings of a HX ring $\vartheta$.

\section{Proof}

Let $\psi_{1}=\left\{\left\langle A, \lambda^{\mu}(\mathrm{A}), \lambda^{\gamma}(\mathrm{A})\right\rangle / \mathrm{A} \in \vartheta\right\}$ and $\psi_{2}=\left\{\left\langle\mathrm{A}, \Theta^{\mu}(\mathrm{A}), \Theta^{\gamma}(\mathrm{A})\right\rangle / \mathrm{A} \in \vartheta\right\}$ be two intuitionistic fuzzy HX subrings of a HX ring $\vartheta$.

To Prove that $\psi_{1} \cap \psi_{2}$ is also an intuitionistic fuzzy HX subring of a HX ring $\vartheta$.

For any $\mathrm{A}, \mathrm{B} \in \vartheta$, we have

$$
\text { (i) } \begin{aligned}
\left(\lambda^{\mu} \cap \Theta^{\mu}\right)(\mathrm{A}-\mathrm{B})=\min \left\{\lambda^{\mu}(\mathrm{A}-\mathrm{B}), \Theta^{\mu}(\mathrm{A}-\mathrm{B})\right\} \\
\geq \min \left\{\min \left\{\lambda^{\mu}(\mathrm{A}), \lambda^{\mu}(\mathrm{B})\right\}, \min \left\{\Theta^{\mu}(\mathrm{A}), \Theta^{\mu}(\mathrm{B})\right\}\right. \\
=\min \left\{\min \left\{\lambda^{\mu}(\mathrm{A}), \Theta^{\mu}(\mathrm{A})\right\}, \min \left\{\lambda^{\mu}(\mathrm{B}), \Theta^{\mu}(\mathrm{B})\right\}\right. \\
=\min \left\{\left(\lambda^{\mu} \cap \Theta^{\mu}\right)(\mathrm{A}),\left(\lambda^{\mu} \cap \Theta^{\mu}\right)(\mathrm{B})\right\}
\end{aligned}
$$

Hence, $\quad\left(\lambda^{\mu} \cap \Theta^{\mu}\right)(A-B) \geq \min \left\{\left(\lambda^{\mu} \cap \Theta^{\mu}\right)(A),\left(\lambda^{\mu} \cap \Theta^{\mu}\right)(B)\right\}$.

$$
\begin{aligned}
\left(\lambda^{\mu}\right. & \left.\cap \Theta^{\mu}\right)(\mathrm{AB})=\min \left\{\lambda^{\mu}(\mathrm{AB}), \Theta^{\mu}(\mathrm{AB})\right\} \\
& \geq \min \left\{\min \left\{\lambda^{\mu}(\mathrm{A}), \lambda^{\mu}(\mathrm{B})\right\}, \min \left\{\Theta^{\mu}(\mathrm{A}), \Theta^{\mu}(\mathrm{B})\right\}\right. \\
& =\min \left\{\min \left\{\lambda^{\mu}(\mathrm{A}), \Theta^{\mu}(\mathrm{A})\right\}, \min \left\{\lambda^{\mu}(\mathrm{B}), \Theta^{\mu}(\mathrm{B})\right\}\right. \\
& =\min \left\{\left(\lambda^{\mu} \cap \Theta^{\mu}\right)(\mathrm{A}),\left(\lambda^{\mu} \cap \Theta^{\mu}\right)(\mathrm{B})\right\}
\end{aligned}
$$

$$
\left(\lambda^{\mu} \cap \Theta^{\mu}\right)(\mathrm{AB}) \geq \min \left\{\left(\lambda^{\mu} \cap \Theta^{\mu}\right)(\mathrm{A}),\left(\lambda^{\mu} \cap \Theta^{\mu}\right)(\mathrm{B})\right\} \text {. }
$$

$$
\begin{aligned}
\left(\lambda^{\gamma} \cap \Theta^{\gamma}\right)(\mathrm{A}-\mathrm{B})=\max \left\{\lambda^{\gamma}(\mathrm{A}-\mathrm{B}), \Theta^{\gamma}(\mathrm{A}-\mathrm{B})\right\} \\
\quad \leq \max \left\{\max \left\{\lambda^{\gamma}(\mathrm{A}), \lambda^{\gamma}(\mathrm{B})\right\}, \max \left\{\Theta^{\gamma}(\mathrm{A}), \Theta^{\gamma}(\mathrm{B})\right\}\right. \\
\quad=\max \left\{\max \left\{\lambda^{\gamma}(\mathrm{A}), \Theta^{\gamma}(\mathrm{A})\right\}, \max \left\{\lambda^{\gamma}(\mathrm{B}), \Theta^{\gamma}(\mathrm{B})\right\}\right. \\
\quad=\max \left\{\left(\lambda^{\gamma} \cap \Theta^{\gamma}\right)(\mathrm{A}),\left(\lambda^{\gamma} \cap \Theta^{\gamma}\right)(\mathrm{B})\right\}
\end{aligned}
$$

Hence, $\quad\left(\lambda^{\gamma} \cap \Theta^{\gamma}\right)(\mathrm{A}-\mathrm{B}) \leq \max \left\{\left(\lambda^{\gamma} \cap \Theta^{\gamma}\right)(\mathrm{A}),\left(\lambda^{\gamma} \cap \Theta^{\gamma}\right)(\mathrm{B})\right\}$.

$$
\begin{aligned}
\left(\lambda^{\gamma}\right. & \left.\cap \Theta^{\gamma}\right)(\mathrm{AB})=\max \left\{\lambda^{\gamma}(\mathrm{AB}), \Theta^{\gamma}(\mathrm{AB})\right\} \\
& \leq \max \left\{\max \left\{\lambda^{\gamma}(\mathrm{A}), \lambda^{\gamma}(\mathrm{B})\right\}, \max \left\{\Theta^{\gamma}(\mathrm{A}), \Theta^{\gamma}(\mathrm{B})\right\}\right. \\
& =\max \left\{\max \left\{\lambda^{\gamma}(\mathrm{A}), \Theta^{\gamma}(\mathrm{A})\right\}, \max \left\{\lambda^{\gamma}(\mathrm{B}), \Theta^{\gamma}(\mathrm{B})\right\}\right. \\
& =\max \left\{\left(\lambda^{\gamma} \cap \Theta^{\gamma}\right)(\mathrm{A}),\left(\lambda^{\gamma} \cap \Theta^{\gamma}\right)(\mathrm{B})\right\}
\end{aligned}
$$

Hence, $\quad\left(\lambda^{\gamma} \cap \Theta^{\gamma}\right)(\mathrm{AB}) \leq \max \left\{\left(\lambda^{\gamma} \cap \Theta^{\gamma}\right)(\mathrm{A}),\left(\lambda^{\gamma} \cap \Theta^{\gamma}\right)(\mathrm{B})\right\}$.

Therefore the intersection of any two IFHX subrings is also an IFHX subring of $\vartheta$. 


\subsection{Theorem}

Let $\psi$ be an intuitionistic fuzzy HX subring of a HX ring $\vartheta$ if and only if $\psi^{\mathrm{c}}$ is an intuitionistic anti fuzzy HX subring of a HX ring $\vartheta$.

\section{Proof}

Let $\psi=\left\{\left\langle\mathrm{A}, \lambda^{\mu}(\mathrm{A}), \lambda^{\gamma}(\mathrm{A})\right\rangle / \mathrm{A} \in \vartheta\right\}$ be a intuitionistic fuzzy HX subring of $\vartheta$.

To prove that $\psi^{\mathrm{c}}$ is an intuitionistic anti fuzzy HX subring of $\vartheta$.

For any $\mathrm{A}, \mathrm{B} \in \vartheta$, we have

(i)

$$
\begin{aligned}
& \Leftrightarrow 1-\left(\lambda^{\mu}\right)^{\mathrm{c}}(\mathrm{A}-\mathrm{B}) \geq \min \left\{1-\left(\lambda^{\mu}\right)^{\mathrm{c}}(\mathrm{A}), 1-\left(\lambda^{\mu}\right)^{\mathrm{c}}(\mathrm{B})\right\} \\
& \Leftrightarrow \quad\left(\lambda^{\mu}\right)^{\mathrm{c}}(\mathrm{A}-\mathrm{B}) \quad \leq 1-\min \left\{1-\left(\lambda^{\mu}\right)^{\mathrm{c}}(\mathrm{A}), 1-\left(\lambda^{\mu}\right)^{\mathrm{c}}(\mathrm{B})\right\} \\
& \Leftrightarrow \quad\left(\lambda^{\mu}\right)^{\mathrm{c}}(\mathrm{A}-\mathrm{B}) \quad \leq \max \left\{\left(\lambda^{\mu}\right)^{\mathrm{c}}(\mathrm{A}),\left(\lambda^{\mu}\right)^{\mathrm{c}}(\mathrm{B})\right\}
\end{aligned}
$$

(ii) $\quad \lambda^{\mu}(\mathrm{AB}) \quad \geq \min \left\{\lambda^{\mu}(\mathrm{A}), \lambda^{\mu}(\mathrm{B})\right\}$

$\Leftrightarrow 1-\left(\lambda^{\mu}\right)^{\mathrm{c}}(\mathrm{AB}) \quad \geq \min \left\{1-\left(\lambda^{\mu}\right)^{\mathrm{c}}(\mathrm{A}), 1-\left(\lambda^{\mu}\right)^{\mathrm{c}}(\mathrm{B})\right\}$

$\Leftrightarrow \quad\left(\lambda^{\mu}\right)^{\mathrm{c}}(\mathrm{AB}) \quad \leq 1-\min \left\{1-\left(\lambda^{\mu}\right)^{\mathrm{c}}(\mathrm{A}), 1-\left(\lambda^{\mu}\right)^{\mathrm{c}}(\mathrm{B})\right\}$

$\Leftrightarrow \quad\left(\lambda^{\mu}\right)^{\mathrm{c}}(\mathrm{AB}) \quad \leq \max \left\{\left(\lambda^{\mu}\right)^{\mathrm{c}}(\mathrm{A}),\left(\lambda^{\mu}\right)^{\mathrm{c}}(\mathrm{B})\right\}$

(iii) $\quad \lambda^{\gamma}(\mathrm{A}-\mathrm{B}) \leq \max \left\{\lambda^{\gamma}(\mathrm{A}), \lambda^{\gamma}(\mathrm{B})\right\}$

$\Leftrightarrow 1-\left(\lambda^{\gamma}\right)^{\mathrm{c}}(\mathrm{A}-\mathrm{B}) \leq \max \left\{1-\left(\lambda^{\gamma}\right)^{\mathrm{c}}(\mathrm{A}), 1-\left(\lambda^{\gamma}\right)^{\mathrm{c}}(\mathrm{B})\right\}$

$\Leftrightarrow \quad\left(\lambda^{\gamma}\right)^{\mathrm{c}}(\mathrm{A}-\mathrm{B}) \quad \geq 1-\max \left\{1-\left(\lambda^{\gamma}\right)^{\mathrm{c}}(\mathrm{A}), 1-\left(\lambda^{\gamma}\right)^{\mathrm{c}}(\mathrm{B})\right\}$

$\Leftrightarrow \quad\left(\lambda^{\gamma}\right)^{\mathrm{c}}(\mathrm{A}-\mathrm{B}) \geq \min \left\{\left(\lambda^{\gamma}\right)^{\mathrm{c}}(\mathrm{A}),\left(\lambda^{\gamma}\right)^{\mathrm{c}}(\mathrm{B})\right\}$.

(iv) $\quad \lambda^{\gamma}(\mathrm{AB}) \leq \max \left\{\lambda^{\gamma}(\mathrm{A}), \lambda^{\gamma}(\mathrm{B})\right\}$

$\Leftrightarrow 1-\left(\lambda^{\gamma}\right)^{\mathrm{c}}(\mathrm{AB}) \leq \max \left\{1-\left(\lambda^{\gamma}\right)^{\mathrm{c}}(\mathrm{A}), 1-\left(\lambda^{\gamma}\right)^{\mathrm{c}}(\mathrm{B})\right\}$

$\Leftrightarrow \quad\left(\lambda^{\gamma}\right)^{\mathrm{c}}(\mathrm{AB}) \quad \geq 1-\max \left\{1-\left(\lambda^{\gamma}\right)^{\mathrm{c}}(\mathrm{A}), 1-\left(\lambda^{\gamma}\right)^{\mathrm{c}}(\mathrm{B})\right\}$

$\Leftrightarrow \quad\left(\lambda^{\gamma}\right)^{\mathrm{c}}(\mathrm{AB}) \quad \geq \min \left\{\left(\lambda^{\gamma}\right)^{\mathrm{c}}(\mathrm{A}),\left(\lambda^{\gamma}\right)^{\mathrm{c}}(\mathrm{B})\right\}$.

Hence $\psi^{\mathrm{c}}=\left\{\left\langle\mathrm{A},\left(\lambda^{\mu}\right)^{\mathrm{c}}(\mathrm{A}),\left(\lambda^{\gamma}\right)^{\mathrm{c}}(\mathrm{A})\right\rangle / \mathrm{A} \in \vartheta\right\}$ is an intuitionistic anti fuzzy HX subring of $\vartheta$.

\subsection{Definition}

Let $\psi=\left\{\left\langle\mathrm{A}, \lambda^{\mu}(\mathrm{A}), \lambda^{\gamma}(\mathrm{A})\right\rangle /\right.$ for all $\left.\mathrm{A} \in \vartheta\right\}$ be an intuitionistic fuzzy subset of a $\mathrm{HX}$ ring $\vartheta$. We define the following "necessity" and "possibility" operations :

$\square \psi=\left\{\left\langle\mathrm{A}, \lambda^{\mu}(\mathrm{A}), 1-\lambda^{\mu}(\mathrm{A})\right\rangle / \mathrm{A} \in \vartheta\right\}$.

$\diamond \psi=\left\{\left\langle\mathrm{A}, 1-\lambda^{\gamma}(\mathrm{A}), \lambda^{\gamma}(\mathrm{A})\right\rangle / \mathrm{A} \in \vartheta\right\}$

\subsection{Theorem}

If $\psi$ is an intuitionistic fuzzy HX subring of a HX ring $\vartheta$ then $\square \psi$ is an intuitionistic fuzzy HX subring of a HX ring $\vartheta$.

\section{Proof}

Let $\square \psi=\left\{\left\langle\mathrm{A}, \lambda^{\mu}(\mathrm{A}),\left(\lambda^{\mu}\right)^{\mathrm{c}}(\mathrm{A})\right\rangle / \mathrm{A} \in \vartheta\right\}$

To prove that $\square \psi$ is an intuitionistic fuzzy HX subring of $\vartheta$.

Let $\psi=\left\{\left\langle\mathrm{A}, \lambda^{\mu}(\mathrm{A}), \lambda^{\gamma}(\mathrm{A})\right\rangle / \mathrm{A} \in \vartheta\right\}$ be a intuitionistic fuzzy HX subring of $\vartheta$. We have

(i) $\quad \lambda^{\mu}(\mathrm{A}-\mathrm{B}) \geq \min \left\{\lambda^{\mu}(\mathrm{A}), \lambda^{\mu}(\mathrm{B})\right\}$,

(ii) $\quad \lambda^{\mu}(\mathrm{AB}) \geq \min \left\{\lambda^{\mu}(\mathrm{A}), \lambda^{\mu}(\mathrm{B})\right\}$,

(iii) $\quad \lambda^{\gamma}(\mathrm{A}-\mathrm{B}) \leq \max \left\{\lambda^{\gamma}(\mathrm{A}), \lambda^{\gamma}(\mathrm{B})\right\}$,

(iv) $\quad \lambda^{\gamma}(\mathrm{AB}) \leq \max \left\{\lambda^{\gamma}(\mathrm{A}), \lambda^{\gamma}(\mathrm{B})\right\}$.

Now

(i) $\left(\lambda^{\mu}\right)^{\mathrm{c}}(\mathrm{A}-\mathrm{B})=1-\left(\lambda^{\mu}\right)(\mathrm{A}-\mathrm{B})$

$\leq 1-\min \left\{\lambda^{\mu}(\mathrm{A}), \lambda^{\mu}(\mathrm{B})\right\}$

$=1-\min \left\{1-\left(\lambda^{\mu}\right)^{\mathrm{c}}(\mathrm{A}), 1-\left(\lambda^{\mu}\right)^{\mathrm{c}}(\mathrm{B})\right\}$

$=\max \left\{\left(\lambda^{\mu}\right)^{\mathrm{c}}(\mathrm{A}),\left(\lambda^{\mu}\right)^{\mathrm{c}}(\mathrm{B})\right\}$

(ii) $\left(\lambda^{\mu}\right)^{\mathrm{c}}(\mathrm{AB})=1-\left(\lambda^{\mu}\right)(\mathrm{AB})$

$\leq 1-\min \left\{\lambda^{\mu}(\mathrm{A}), \lambda^{\mu}(\mathrm{B})\right\}$

$=1-\min \left\{1-\left(\lambda^{\mu}\right)^{\mathrm{c}}(\mathrm{A}), 1-\left(\lambda^{\mu}\right)^{\mathrm{c}}(\mathrm{B})\right\}$

Hence,

$=\max \left\{\left(\lambda^{\mu}\right)^{\mathrm{c}}(\mathrm{A}),\left(\lambda^{\mu}\right)^{\mathrm{c}}(\mathrm{B})\right\}$

$\lambda^{\mu}(\mathrm{A}-\mathrm{B}) \geq \min \left\{\lambda^{\mu}(\mathrm{A}), \lambda^{\mu}(\mathrm{B})\right\}$,

$\lambda^{\mu}(\mathrm{AB}) \geq \min \left\{\lambda^{\mu}(\mathrm{A}), \lambda^{\mu}(\mathrm{B})\right\}$, 
$\left(\lambda^{\mu}\right)^{\mathrm{c}}(\mathrm{A}-\mathrm{B}) \leq \max \left\{\left(\lambda^{\mu}\right)^{\mathrm{c}}(\mathrm{A}),\left(\lambda^{\mu}\right)^{\mathrm{c}}(\mathrm{B})\right\}$ and $\left(\lambda^{\mu}\right)^{\mathrm{c}}(\mathrm{AB}) \leq \max \left\{\left(\lambda^{\mu}\right)^{\mathrm{c}}(\mathrm{A}),\left(\lambda^{\mu}\right)^{\mathrm{c}}(\mathrm{B})\right\}$.

Therefore $\square \psi=\left\{\left\langle A, \lambda^{\mu}(\mathrm{A}),\left(\lambda^{\mu}\right)^{\mathrm{c}}(\mathrm{A})\right\rangle / \mathrm{A} \in \vartheta\right\}$ is a intuitionistic fuzzy HX subring of $\vartheta$.

\subsection{Theorem}

If $\psi$ is an intuitionistic fuzzy HX subring of a HX ring $\vartheta$ then $\diamond \psi$ is an intuitionistic fuzzy HX subring of a HX ring $\vartheta$.

\section{Proof}

Let $\diamond \psi=\left\{\left\langle\mathrm{A},\left(\lambda^{\gamma}\right)^{\mathrm{c}}(\mathrm{A}), \lambda^{\gamma}(\mathrm{A})\right\rangle / \mathrm{A} \in \vartheta\right\}$

To prove that $\diamond \psi$ is an intuitionistic fuzzy HX subring of $\vartheta$.

Let $\psi=\left\{\left\langle\mathrm{A}, \lambda^{\mu}(\mathrm{A}), \lambda^{\gamma}(\mathrm{A})\right\rangle / \mathrm{A} \in \vartheta\right\}$ be a intuitionistic fuzzy HX subring of $\vartheta$. We have

(i) $\quad \lambda^{\mu}(\mathrm{A}-\mathrm{B}) \geq \min \left\{\lambda^{\mu}(\mathrm{A}), \lambda^{\mu}(\mathrm{B})\right\}$,

(ii) $\quad \lambda^{\mu}(\mathrm{AB}) \geq \min \left\{\lambda^{\mu}(\mathrm{A}), \lambda^{\mu}(\mathrm{B})\right\}$,

(iii) $\quad \lambda^{\gamma}(\mathrm{A}-\mathrm{B}) \leq \max \left\{\lambda^{\gamma}(\mathrm{A}), \lambda^{\gamma}(\mathrm{B})\right\}$,

(iv) $\quad \lambda^{\gamma}(\mathrm{AB}) \leq \max \left\{\lambda^{\gamma}(\mathrm{A}), \lambda^{\gamma}(\mathrm{B})\right\}$.

Now

(i)

$$
\begin{aligned}
& \left(\lambda^{\gamma}\right)^{\mathrm{c}}(\mathrm{A}-\mathrm{B})=1-\left(\lambda^{\gamma}\right)(\mathrm{A}-\mathrm{B}) \\
& \geq 1-\max \left\{\lambda^{\gamma}(\mathrm{A}), \lambda^{\gamma}(\mathrm{B})\right\} \\
& =1-\max \left\{1-\left(\lambda^{\gamma}\right)^{\mathrm{c}}(\mathrm{A}), 1-\left(\lambda^{\gamma}\right)^{\mathrm{c}}(\mathrm{B})\right\} \\
& =\min \left\{\left(\lambda^{\gamma}\right)^{\mathrm{c}}(\mathrm{A}),\left(\lambda^{\gamma}\right)^{\mathrm{c}}(\mathrm{B})\right\}
\end{aligned}
$$

(ii)

$$
\begin{aligned}
& \left(\lambda^{\gamma}\right)^{\mathrm{c}}(\mathrm{AB})=1-\left(\lambda^{\gamma}\right)(\mathrm{AB}) \\
& \geq 1-\max \left\{\lambda^{\gamma}(\mathrm{A}), \lambda^{\gamma}(\mathrm{B})\right\} \\
& =1-\max \left\{1-\left(\lambda^{\gamma}\right)^{\mathrm{c}}(\mathrm{A}), 1-\left(\lambda^{\gamma}\right)^{\mathrm{c}}(\mathrm{B})\right\} \\
& \quad=\min \left\{\left(\lambda^{\gamma}\right)^{\mathrm{c}}(\mathrm{A}),\left(\lambda^{\gamma}\right)^{\mathrm{c}}(\mathrm{B})\right\}
\end{aligned}
$$

Hence, $\quad \lambda^{\gamma}(\mathrm{A}-\mathrm{B}) \leq \max \left\{\lambda^{\gamma}(\mathrm{A}), \lambda^{\gamma}(\mathrm{B})\right\}$, $\lambda^{\gamma}(\mathrm{AB}) \leq \max \left\{\lambda^{\gamma}(\mathrm{A}), \lambda^{\gamma}(\mathrm{B})\right\}$. $\left(\lambda^{\gamma}\right)^{\mathrm{c}}(\mathrm{A}-\mathrm{B}) \geq \min \left\{\left(\lambda^{\gamma}\right)^{\mathrm{c}}(\mathrm{A}),\left(\lambda^{\gamma}\right)^{\mathrm{c}}(\mathrm{B})\right\}$ and $\left(\lambda^{\gamma}\right)^{\mathrm{c}}(\mathrm{AB}) \geq \min \left\{\left(\lambda^{\gamma}\right)^{\mathrm{c}}(\mathrm{A}),\left(\lambda^{\gamma}\right)^{\mathrm{c}}(\mathrm{B})\right\}$.

Therefore, $\diamond_{\psi}=\left\{\left\langle\mathrm{A},\left(\lambda^{\gamma}\right)^{\mathrm{c}}(\mathrm{A}), \lambda^{\gamma}(\mathrm{A})\right\rangle / \mathrm{A} \in \vartheta\right\}$ is a intuitionistic fuzzy HX subring of $\vartheta$.

\subsection{Theorem}

An IFS $\psi=\left\{\left\langle A, \lambda^{\mu}(\mathrm{A}), \lambda^{\gamma}(\mathrm{A})\right\rangle / \mathrm{A} \in \vartheta\right\}$ is an intuitionistic fuzzy $\mathrm{HX}$ subring of a $\mathrm{HX}$ ring $\vartheta$ if and only if the fuzzy subsets $\lambda^{\mu}(\mathrm{A}),\left(\lambda^{\gamma}\right)^{\mathrm{c}}(\mathrm{A})$ are fuzzy HX subring of a HX ring $\vartheta$.

\section{Proof}

Let $\psi=\left\{\left\langle A, \lambda^{\mu}(\mathrm{A}), \lambda^{\gamma}(\mathrm{A})\right\rangle / \mathrm{A} \in \vartheta\right\}$ be a intuitionistic fuzzy HX subring of $\vartheta$. We have

(i) $\quad \lambda^{\mu}(\mathrm{A}-\mathrm{B}) \geq \min \left\{\lambda^{\mu}(\mathrm{A}), \lambda^{\mu}(\mathrm{B})\right\}$,

(ii) $\quad \lambda^{\mu}(\mathrm{AB}) \geq \min \left\{\lambda^{\mu}(\mathrm{A}), \lambda^{\mu}(\mathrm{B})\right\}$,

(iii) $\quad \lambda^{\gamma}(\mathrm{A}-\mathrm{B}) \leq \max \left\{\lambda^{\gamma}(\mathrm{A}), \lambda^{\gamma}(\mathrm{B})\right\}$,

(iv) $\quad \lambda^{\gamma}(\mathrm{AB}) \leq \max \left\{\lambda^{\gamma}(\mathrm{A}), \lambda^{\gamma}(\mathrm{B})\right\}$.

Clearly, $\lambda^{\mu}(\mathrm{A})$ is a fuzzy HX subring of $\vartheta$ by (i) and (ii) .

Now we have to show $\left(\lambda^{\gamma}\right)^{c}$ is a fuzzy HX subring of $\vartheta$.

(i) $\left(\lambda^{\gamma}\right)^{\mathrm{c}}(\mathrm{A}-\mathrm{B})=1-\left(\lambda^{\gamma}\right)(\mathrm{A}-\mathrm{B})$

$$
\begin{aligned}
& \geq 1-\max \left\{\lambda^{\gamma}(\mathrm{A}), \lambda^{\gamma}(\mathrm{B})\right\} \\
& =1-\max \left\{1-\left(\lambda^{\gamma}\right)^{\mathrm{c}}(\mathrm{A}), 1-\left(\lambda^{\gamma}\right)^{\mathrm{c}}(\mathrm{B})\right\} \\
& \quad=\min \left\{\left(\lambda^{\gamma}\right)^{\mathrm{c}}(\mathrm{A}),\left(\lambda^{\gamma}\right)^{\mathrm{c}}(\mathrm{B})\right\} \\
& \left(\lambda^{\gamma}\right)^{\mathrm{c}}(\mathrm{A}-\mathrm{B}) \geq \min \left\{\left(\lambda^{\gamma}\right)^{\mathrm{c}}(\mathrm{A}),\left(\lambda^{\gamma}\right)^{\mathrm{c}}(\mathrm{B})\right\}
\end{aligned}
$$

(ii) $\left(\lambda^{\gamma}\right)^{\mathrm{c}}(\mathrm{AB})=1-\left(\lambda^{\gamma}\right)(\mathrm{AB})$

$$
\begin{aligned}
& \geq 1-\max \left\{\lambda^{\gamma}(\mathrm{A}), \lambda^{\gamma}(\mathrm{B})\right\} \\
& =1-\max \left\{1-\left(\lambda^{\gamma}\right)^{\mathrm{c}}(\mathrm{A}), 1-\left(\lambda^{\gamma}\right)^{\mathrm{c}}(\mathrm{B})\right\} \\
& \quad=\min \left\{\left(\lambda^{\gamma}\right)^{\mathrm{c}}(\mathrm{A}),\left(\lambda^{\gamma}\right)^{\mathrm{c}}(\mathrm{B})\right\} \\
& \left(\lambda^{\gamma}\right)^{\mathrm{c}}(\mathrm{AB}) \geq \min \left\{\left(\lambda^{\gamma}\right)^{\mathrm{c}}(\mathrm{A}),\left(\lambda^{\gamma}\right)^{\mathrm{c}}(\mathrm{B})\right\} .
\end{aligned}
$$

Conversely,

Thus $\left(\lambda^{\gamma}\right)^{c}$ is a fuzzy HX subring of $\vartheta$.

$\lambda^{\mu}(\mathrm{A})$ and $\left(\lambda^{\gamma}\right)^{\mathrm{c}}(\mathrm{A})$ are fuzzy HX subring of a HX ring $\vartheta$

To prove that $\psi=\left\{\left\langle\mathrm{A}, \lambda^{\mu}(\mathrm{A}), \lambda^{\gamma}(\mathrm{A})\right\rangle / \mathrm{A} \in \vartheta\right\}$ be a intuitionistic fuzzy HX subring of $\vartheta$.

Now we know that 


$$
\begin{aligned}
\left(\lambda^{\gamma}\right)^{\mathrm{c}}(\mathrm{A}-\mathrm{B}) & \geq \min \left\{\left(\lambda^{\gamma}\right)^{\mathrm{c}}(\mathrm{A}),\left(\lambda^{\gamma}\right)^{\mathrm{c}}(\mathrm{B})\right\} \\
1-\left(\lambda^{\gamma}\right)(\mathrm{A}-\mathrm{B}) & \geq \min \left\{1-\left(\lambda^{\gamma}\right)(\mathrm{A}), 1-\left(\lambda^{\gamma}\right)(\mathrm{B})\right\} . \\
1-\left(\lambda^{\gamma}\right)(\mathrm{A}-\mathrm{B}) & =1-\max \left\{\lambda^{\gamma}(\mathrm{A}), \lambda^{\gamma}(\mathrm{B})\right\} \text { implies } \\
\left(\lambda^{\gamma}\right)(\mathrm{A}-\mathrm{B}) & \leq \max \left\{\lambda^{\gamma}(\mathrm{A}), \lambda^{\gamma}(\mathrm{B})\right\}
\end{aligned}
$$

Also

$$
\begin{aligned}
\left(\lambda^{\gamma}\right)^{\mathrm{c}}(\mathrm{AB}) & \geq \min \left\{\left(\lambda^{\gamma}\right)^{\mathrm{c}}(\mathrm{A}),\left(\lambda^{\gamma}\right)^{\mathrm{c}}(\mathrm{B})\right\} \\
1-\left(\lambda^{\gamma}\right)(\mathrm{AB}) & \geq \min \left\{1-\left(\lambda^{\gamma}\right)(\mathrm{A}), 1-\left(\lambda^{\gamma}\right)(\mathrm{B})\right\} .
\end{aligned}
$$$$
1-\left(\lambda^{\gamma}\right)(\mathrm{AB})=1-\max \left\{\lambda^{\gamma}(\mathrm{A}), \lambda^{\gamma}(\mathrm{B})\right\} \text { implies }
$$$$
\left(\lambda^{\gamma}\right)(\mathrm{AB}) \leq \max \left\{\lambda^{\gamma}(\mathrm{A}), \lambda^{\gamma}(\mathrm{B})\right\}
$$

Already we have

$$
\lambda^{\mu}(\mathrm{A}-\mathrm{B}) \geq \min \left\{\lambda^{\mu}(\mathrm{A}), \lambda^{\mu}(\mathrm{B})\right\}, \quad \lambda^{\mu}(\mathrm{AB}) \geq \min \left\{\lambda^{\mu}(\mathrm{A}), \lambda^{\mu}(\mathrm{B})\right\} .
$$

Hence $\quad \psi=\left\{\left\langle\mathrm{A}, \lambda^{\mu}(\mathrm{A}), \lambda^{\gamma}(\mathrm{A})\right\rangle / \mathrm{A} \in \vartheta\right\}$ be a intuitionistic fuzzy HX subring of $\vartheta$.

\subsection{Theorem}

An IFS $\psi=\left\{\left\langle\mathrm{A}, \lambda^{\mu}(\mathrm{A}), \lambda^{\gamma}(\mathrm{A})\right\rangle / \mathrm{A} \in \vartheta\right\}$ is an intuitionistic fuzzy HX subring of a HX ring $\vartheta$ if and only if the fuzzy subsets $\left(\lambda^{\mu}\right)^{\mathrm{c}}$ and $\left(\lambda^{\gamma}\right)$ are anti-fuzzy HX subring of a HX ring $\vartheta$.

\section{Proof}

Let $\psi=\left\{\left\langle\mathrm{A}, \lambda^{\mu}(\mathrm{A}), \lambda^{\gamma}(\mathrm{A})\right\rangle / \mathrm{A} \in \vartheta\right\}$ be a intuitionistic fuzzy HX subring of $\vartheta$. We have

(i) $\quad \lambda^{\mu}(\mathrm{A}-\mathrm{B}) \geq \min \left\{\lambda^{\mu}(\mathrm{A}), \lambda^{\mu}(\mathrm{B})\right\}$,

(ii) $\quad \lambda^{\mu}(\mathrm{AB}) \geq \min \left\{\lambda^{\mu}(\mathrm{A}), \lambda^{\mu}(\mathrm{B})\right\}$,

(iii) $\quad \lambda^{\gamma}(\mathrm{A}-\mathrm{B}) \leq \max \left\{\lambda^{\gamma}(\mathrm{A}), \lambda^{\gamma}(\mathrm{B})\right\}$,

(iv) $\quad \lambda^{\gamma}(\mathrm{AB}) \leq \max \left\{\lambda^{\gamma}(\mathrm{A}), \lambda^{\gamma}(\mathrm{B})\right\}$.

From (iii) and (iv) it is clear that $\left(\lambda^{\gamma}\right)$ is an anti-fuzzy HX subring of $\vartheta$. Now ,

$$
\begin{aligned}
\left(\lambda^{\mu}\right)^{\mathrm{c}}(\mathrm{A}-\mathrm{B}) & =1-\left(\lambda^{\mu}\right)(\mathrm{A}-\mathrm{B}) \\
& \leq 1-\min \left\{\lambda^{\mu}(\mathrm{A}), \lambda^{\mu}(\mathrm{B})\right\} \\
& =1-\min \left\{1-\left(\lambda^{\mu}\right)^{\mathrm{c}}(\mathrm{A}), 1-\left(\lambda^{\mu}\right)^{\mathrm{c}}(\mathrm{B})\right\} \\
& =\max \left\{\left(\lambda^{\mu}\right)^{\mathrm{c}}(\mathrm{A}),\left(\lambda^{\mu}\right)^{\mathrm{c}}(\mathrm{B})\right\} \\
\left(\lambda^{\mu}\right)^{\mathrm{c}}(\mathrm{A}-\mathrm{B}) & \leq \max \left\{\left(\lambda^{\mu}\right)^{\mathrm{c}}(\mathrm{A}),\left(\lambda^{\mu}\right)^{\mathrm{c}}(\mathrm{B})\right\} . \\
& \begin{aligned}
\left(\lambda^{\mu}\right)^{\mathrm{c}}(\mathrm{AB}) & =1-\left(\lambda^{\mu}\right)(\mathrm{AB}) \\
& \leq 1-\min \left\{\lambda^{\mu}(\mathrm{A}), \lambda^{\mu}(\mathrm{B})\right\} \\
& =1-\min \left\{1-\left(\lambda^{\mu}\right)^{\mathrm{c}}(\mathrm{A}), 1-\left(\lambda^{\mu}\right)^{\mathrm{c}}(\mathrm{B})\right\} \\
& =\max \left\{\left(\lambda^{\mu}\right)^{\mathrm{c}}(\mathrm{A}),\left(\lambda^{\mu}\right)^{\mathrm{c}}(\mathrm{B})\right\} \\
\left(\lambda^{\mu}\right)^{\mathrm{c}}(\mathrm{AB}) & \leq \max \left\{\left(\lambda^{\mu}\right)^{\mathrm{c}}(\mathrm{A}),\left(\lambda^{\mu}\right)^{\mathrm{c}}(\mathrm{B})\right\} .
\end{aligned}
\end{aligned}
$$
Also

Hence, $\left(\lambda^{\mu}\right)^{c}$ and $\left(\lambda^{\gamma}\right)$ are anti-fuzzy HX subring of a HX ring $\vartheta$.

\section{Conversely,}

$\left(\lambda^{\mu}\right)^{c}$ and $\left(\lambda^{\gamma}\right)$ are anti-fuzzy HX subring of a HX ring $\vartheta$

To prove that $\psi=\left\{\left\langle\mathrm{A}, \lambda^{\mu}(\mathrm{A}), \lambda^{\gamma}(\mathrm{A})\right\rangle / \mathrm{A} \in \vartheta\right\}$ be a intuitionistic fuzzy HX subring of $\vartheta$.

It is clear that $\lambda^{\gamma}(\mathrm{A}-\mathrm{B}) \leq \max \left\{\lambda^{\gamma}(\mathrm{A}), \lambda^{\gamma}(\mathrm{B})\right\}$ and

Now

$$
\lambda^{\gamma}(\mathrm{AB}) \leq \max \left\{\lambda^{\gamma}(\mathrm{A}), \lambda^{\gamma}(\mathrm{B})\right\} .
$$

$$
\begin{aligned}
\left(\lambda^{\mu}\right)^{\mathrm{c}}(\mathrm{A}-\mathrm{B}) & \leq \max \left\{\left(\lambda^{\mu}\right)^{\mathrm{c}}(\mathrm{A}),\left(\lambda^{\mu}\right)^{\mathrm{c}}(\mathrm{B})\right\} \\
1-\left(\lambda^{\mu}\right)(\mathrm{A}-\mathrm{B}) & =\max \left\{1-\left(\lambda^{\mu}\right)(\mathrm{A}), 1-\left(\lambda^{\mu}\right)(\mathrm{B})\right\} . \\
1-\left(\lambda^{\mu}\right)(\mathrm{A}-\mathrm{B}) & =1-\min \left\{\lambda^{\mu}(\mathrm{A}), \lambda^{\mu}(\mathrm{B})\right\} \text { implies } \\
\left(\lambda^{\mu}\right)(\mathrm{A}-\mathrm{B}) & \geq \min \left\{\lambda^{\mu}(\mathrm{A}), \lambda^{\mu}(\mathrm{B})\right\} .
\end{aligned}
$$

Also

$$
\begin{aligned}
\left(\lambda^{\mu}\right)^{\mathrm{c}}(\mathrm{AB}) & \leq \max \left\{\left(\lambda^{\mu}\right)^{\mathrm{c}}(\mathrm{A}),\left(\lambda^{\mu}\right)^{\mathrm{c}}(\mathrm{B})\right\} \\
1-\left(\lambda^{\mu}\right)(\mathrm{AB}) & =\max \left\{1-\left(\lambda^{\mu}\right)(\mathrm{A}), 1-\left(\lambda^{\mu}\right)(\mathrm{B})\right\} . \\
1-\left(\lambda^{\mu}\right)(\mathrm{AB}) & =1-\min \left\{\lambda^{\mu}(\mathrm{A}), \lambda^{\mu}(\mathrm{B})\right\} \text { implies } \\
\left(\lambda^{\mu}\right)(\mathrm{AB}) & \geq \min \left\{\lambda^{\mu}(\mathrm{A}), \lambda^{\mu}(\mathrm{B})\right\} .
\end{aligned}
$$

Thus $\quad \lambda^{\mu}(\mathrm{A}-\mathrm{B}) \geq \min \left\{\lambda^{\mu}(\mathrm{A}), \lambda^{\mu}(\mathrm{B})\right\}, \quad \lambda^{\mu}(\mathrm{AB}) \geq \min \left\{\lambda^{\mu}(\mathrm{A}), \lambda^{\mu}(\mathrm{B})\right\}$,

$\lambda^{\gamma}(\mathrm{A}-\mathrm{B}) \leq \max \left\{\lambda^{\gamma}(\mathrm{A}), \lambda^{\gamma}(\mathrm{B})\right\}$ and $\lambda^{\gamma}(\mathrm{AB}) \leq \max \left\{\lambda^{\gamma}(\mathrm{A}), \lambda^{\gamma}(\mathrm{B})\right\}$.

Hence $\psi=\left\{\left\langle\mathrm{A}, \lambda^{\mu}(\mathrm{A}), \lambda^{\gamma}(\mathrm{A})\right\rangle / \mathrm{A} \in \vartheta\right\}$ be a intuitionistic fuzzy HX subring of $\vartheta$. 


\subsection{Definition}

Let $\vartheta_{1}$ and $\vartheta_{2}$ be any two HX rings. Then the function $f: \vartheta_{1} \rightarrow \vartheta_{2}$ is said to be a homomorphism if it satisfies the following axioms:
i) $f(A+B)=f(A)+f(B)$ and
ii) $f(A B)=f(A) f(B)$, for all $A, B \in \vartheta_{1}$

\subsection{Definition}

Let $\vartheta_{1}$ and $\vartheta_{2}$ be any two HX rings. Then the function $f: \vartheta_{1} \rightarrow \vartheta_{2}$ is said to be an anti homomorphism if it satisfies the following axioms:
i) $f(A+B)=f(B)+f(A)$ and
ii) $\mathrm{f}(\mathrm{AB})=\mathrm{f}(\mathrm{B}) \mathrm{f}(\mathrm{A})$, for all $\mathrm{A}, \mathrm{B} \in \vartheta_{1}$.

\subsection{Definition}

Let $R_{1}$ and $R_{2}$ be any two rings. Let $\vartheta_{1} \subset 2^{R 1}-\{\phi\}$ and $\vartheta_{2} \subset 2^{R 2}-\{\phi\}$ be any two HX rings. Let $A=$ $\left\{\left(\mathrm{x}, \mu_{\mathrm{A}}(\mathrm{x}), \gamma_{\mathrm{A}}(\mathrm{x})\right) / \mathrm{x} \in \mathrm{R}_{1}\right\}$ and $\mathrm{B}=\left\{\left(\mathrm{y}, \alpha_{\mathrm{B}}(\mathrm{y}), \beta_{\mathrm{B}}(\mathrm{y})\right) / \mathrm{y} \in \mathrm{R}_{2}\right\}$ be any two intuitionistic fuzzy sets on $\mathrm{R}_{1}$ and $\mathrm{R}_{2}$ respectively. Let $\mathrm{C}=\left\{\left(\mathrm{U}, \lambda_{C}^{\mu}(U), \theta_{C}^{\gamma}(U)\right) / \mathrm{U} \in \vartheta_{1}\right\}$ and $\mathrm{D}=\left\{\left(\mathrm{V}, \eta_{D}^{\alpha}(V), \psi_{D}^{\beta}(V)\right) / \mathrm{V} \in \vartheta_{2}\right\}$ any two intuitionistic fuzzy sets in $\vartheta_{1}$ and $\vartheta_{2}$ resp. Let $\mathrm{f}$ be a function from $\vartheta_{1}$ into $\vartheta_{2}$ then the image of $\mathrm{C}$ on $\vartheta_{1}$ under $f$ is defined as

$$
\eta_{D}^{\alpha}(V)=\left\{\begin{array}{l}
\max \left\{\lambda_{C}^{\mu}(U): U \in f^{-1}(V)\right\}, f^{-1}(V) \neq \phi \\
0 \quad, \text { otherwise }
\end{array} \quad \text { and } \quad \psi_{D}^{\beta}(V)=\left\{\begin{array}{l}
\min \left\{\theta_{C}^{\gamma}(U): U \in f^{-1}(V)\right\}, f^{-1}(V) \neq \phi \\
1 \quad \text { otherwise }
\end{array}\right.\right.
$$

Where $\eta_{D}^{\alpha}=f\left(\lambda_{C}^{\mu}\right)$ also Pre-image of $\mathrm{D}$ on $\vartheta_{2}$ under $\mathrm{f}$ is defined as $\left(f^{-1}\left(\eta_{D}^{\alpha}\right)\right)(U)=\eta_{D}^{\alpha}(f(U))$, $\left(f^{-1}\left(\psi_{D}^{\beta}\right)\right)(U)=\psi_{D}^{\beta}(f(U))$.

\subsection{Theorem}

Let $R_{1}$ and $R_{2}$ be any two rings. Let $A=\left\{\left(x, \mu_{A}(x), \gamma_{A}(x)\right) / x \in R_{1}\right\}$ and $\mathrm{B}=\left\{(\mathrm{y}, \alpha \mathrm{B}(\mathrm{y}), \beta \mathrm{B}(\mathrm{y})) / \mathrm{y} \in \mathrm{R}_{2}\right\}$ be any two intuitionistic fuzzy sets on $\mathrm{R}_{1}$ and $\mathrm{R}_{2}$ respectively. Let $\mathrm{C}=\left\{\left(\mathrm{U}, \lambda_{C}^{\mu}(U), \theta_{C}^{\gamma}(U)\right) / \mathrm{U} \in \vartheta_{1}\right\}$ and $\mathrm{D}=\left\{\left(\mathrm{V}, \eta_{D}^{\alpha}(V), \psi_{D}^{\beta}(V)\right) / \mathrm{V} \in \vartheta_{2}\right\}$ be any two intuitionistic fuzzy sets in $\vartheta_{1}$ and $\vartheta_{2}$ resp. Let $\mathrm{f}$ be a onto homomorphism from $\vartheta_{1}$ to $\vartheta_{2}$. If $\mathrm{C}$ be the intuitionistic fuzzy HX subring of $\vartheta_{1}$ then $\mathrm{f}(\mathrm{C})$ is a intuitionistic fuzzy HX subring of $\vartheta_{2}$.

Proof.

Let $\mathrm{C}$ be the intuitionistic fuzzy HX subring of $\vartheta_{1}$ then

$$
\begin{array}{ll}
\text { (i) } & \lambda_{C}^{\mu}(U-T) \geq \min \left\{\lambda_{C}^{\mu}(U), \lambda_{C}^{\mu}(T)\right\} \\
\text { (ii) } & \lambda_{C}^{\mu}(U T) \geq \min \left\{\lambda_{C}^{\mu}(U), \lambda_{C}^{\mu}(T)\right\} \\
\text { (iii) } & \theta_{C}^{\gamma}(U-T) \leq \max \left\{\lambda_{C}^{\mu}(U), \lambda_{C}^{\mu}(T)\right\} \\
\text { (iv) } & \theta_{C}^{\gamma}(U T) \leq \max \left\{\lambda_{C}^{\mu}(U), \lambda_{C}^{\mu}(T)\right\}
\end{array}
$$

To Prove that $\mathrm{f}(\mathrm{C})$ is an intuitionistic fuzzy HX subring of $\vartheta_{2}$. Let $\mathrm{V}=\mathrm{f}(\mathrm{U}), \mathrm{W}=\mathrm{f}(\mathrm{T}) \in \vartheta_{2}$, where $\mathrm{U}, \mathrm{T} \in \vartheta_{1}$.

$$
\text { Now } \begin{array}{rlr}
\eta_{D}^{\alpha}[f(U)-f(T)]=\eta_{D}^{\alpha}[f(U-T)] & (\mathrm{f} \text { is homomorphism }) \\
= & \lambda_{C}^{\mu}[U-T] \quad(\mathrm{f} \text { is onto }) \\
\geq \min \left\{\lambda_{C}^{\mu}(U), \lambda_{C}^{\mu}(T)\right\} & \\
& \geq \min \left\{\eta_{D}^{\alpha}(f(U)), \eta_{D}^{\alpha}(f(T))\right\}
\end{array}
$$

Hence, $\quad \eta_{D}^{\alpha}[f(U)-f(T)] \geq \min \left\{\eta_{D}^{\alpha}(f(U)), \eta_{D}^{\alpha}(f(T))\right\}$

Again $\quad \eta_{D}^{\alpha}[f(U) f(T)]=\eta_{D}^{\alpha}[f(U T)] \quad(\mathrm{f}$ is homomorphism $)$

$$
\begin{aligned}
& =\lambda_{C}^{\mu}[U T] \quad(\mathrm{f} \text { is onto }) \\
& \geq \min \left\{\lambda_{C}^{\mu}(U), \lambda_{C}^{\mu}(T)\right\} \\
& \geq \min \left\{\eta_{D}^{\alpha}(f(U)), \eta_{D}^{\alpha}(f(T))\right\}
\end{aligned}
$$

Hence, $\quad \eta_{D}^{\alpha}[f(U) f(T)] \geq \min \left\{\eta_{D}^{\alpha}(f(U)), \eta_{D}^{\alpha}(f(T))\right\}$

Now 


$$
\begin{aligned}
\psi_{D}^{\beta}[f(U)-f(T)] & =\psi_{D}^{\beta}[f(U-T)] \quad(\mathrm{f} \text { is homomorphism }) \\
& =\theta_{C}^{\gamma}[U-T] \quad(\mathrm{f} \text { is onto }) \\
& \leq \max \left\{\theta_{C}^{\gamma}(U), \theta_{C}^{\gamma}(T)\right\} \\
& =\max \left\{\psi_{D}^{\beta}(f(U)), \psi_{D}^{\beta}(f(T))\right\}
\end{aligned}
$$

Hence, $\quad \psi_{D}^{\beta}[f(U)-f(T)] \leq \max \left\{\psi_{D}^{\beta}(f(U)), \psi_{D}^{\beta}(f(T))\right\}$

Also

$$
\begin{aligned}
\psi_{D}^{\beta}[f(U) f(T)] & =\psi_{D}^{\beta}[f(U T)] \quad(\mathrm{f} \text { is homomorphism }) \\
& =\theta_{C}^{\gamma}(U T) \quad(\mathrm{f} \text { is onto }) \\
& \leq \max \left\{\theta_{C}^{\gamma}(U), \theta_{C}^{\gamma}(T)\right\} \\
& =\max \left\{\psi_{D}^{\beta}(f(U)), \psi_{D}^{\beta}(f(T))\right\}
\end{aligned}
$$

Hence, $\quad \psi_{D}^{\beta}[f(U T)] \leq \max \left\{\psi_{D}^{\beta}(f(U)), \psi_{D}^{\beta}(f(T))\right\}$

Thus $\mathrm{D}=\mathrm{f}(\mathrm{C})$ is an intuitionistic fuzzy HX subring of $\vartheta_{2}$.

\subsection{Theorem}

Let $\mathrm{R}_{1}$ and $\mathrm{R}_{2}$ be any two rings. Let $\mathrm{A}=\left\{\left(\mathrm{x}, \mu_{\mathrm{A}}(\mathrm{x}), \gamma_{\mathrm{A}}(\mathrm{x})\right) / \mathrm{x} \in \mathrm{R}_{1}\right\}$ and $\mathrm{B}=\left\{\left(\mathrm{y}, \alpha_{\mathrm{B}}(\mathrm{y}), \beta_{\mathrm{B}}(\mathrm{y})\right) / \mathrm{y} \in \mathrm{R}_{2}\right\}$ be any two intuitionistic fuzzy sets on $\mathrm{R}_{1}$ and $\mathrm{R}_{2}$ respectively.

Let $\mathrm{C}=\left\{\left(\mathrm{U}, \lambda_{C}^{\mu}(U), \theta_{C}^{\gamma}(U)\right) / \mathrm{U} \in \vartheta_{1}\right\}$ and $\mathrm{D}=\left\{\left(\mathrm{V}, \eta_{D}^{\alpha}(V), \psi_{D}^{\beta}(V)\right) / \mathrm{V} \in \vartheta_{2}\right\}$ be any two intuitionistic fuzzy sets in $\vartheta_{1}$ and $\vartheta_{2}$ resp. Let $\mathrm{f}$ be an onto homomorphism from $\vartheta_{1}$ to $\vartheta_{2}$. If $\mathrm{D}$ be the intuitionistic fuzzy HX subring of $\vartheta_{2}$ then $\mathrm{f}^{-1}(\mathrm{D})$ is a intuitionistic fuzzy HX subring of $\vartheta_{1}$.

Proof.

Let $\mathrm{D}$ be the intuitionistic fuzzy HX subring of $\vartheta_{2}$ then

$$
\begin{array}{ll}
\text { (i) } & \eta_{D}^{\alpha}[V-W] \geq \min \left\{\eta_{D}^{\alpha}(V), \eta_{D}^{\alpha}(W)\right\} \\
\text { (ii) } & \eta_{D}^{\alpha}[V W] \geq \min \left\{\eta_{D}^{\alpha}(V), \eta_{D}^{\alpha}(W)\right\} \\
\text { (iii) } & \psi_{D}^{\beta}[V-W] \leq \max \left\{\psi_{D}^{\beta}(V), \psi_{D}^{\beta}(W)\right\} \\
\text { (iv) } & \psi_{D}^{\beta}[V W] \leq \max \left\{\psi_{D}^{\beta}(V), \psi_{D}^{\beta}(W)\right\}
\end{array}
$$

To Prove that $\mathrm{f}^{-1}(\mathrm{D})$ is a intuitionistic fuzzy HX subring of $\vartheta_{1}$.

Let $\mathrm{U}, \mathrm{T} \in \vartheta_{1}$ and $\mathrm{f}(\mathrm{U})=\mathrm{V}, \mathrm{f}(\mathrm{T})=\mathrm{W} \in \vartheta_{2}$.

Now

$$
\begin{aligned}
{\left[f^{-1}\left(\eta_{D}^{\alpha}\right)\right](U-T) } & =\eta_{D}^{\alpha}[f(U-T)] \\
& =\eta_{D}^{\alpha}[f(U)-f(T)] \quad(\mathrm{f} \text { is homomorphism }) \\
\geq & \min \left\{\eta_{D}^{\alpha}(f(U)), \eta_{D}^{\alpha}(f(T))\right\} \\
& =\min \left\{\left[f^{-1}\left(\eta_{D}^{\alpha}\right)\right](U),\left[f^{-1}\left(\eta_{D}^{\alpha}\right)\right](T)\right\}
\end{aligned}
$$

Hence, $\quad\left[f^{-1}\left(\eta_{D}^{\alpha}\right)\right](U-T) \geq \min \left\{\left[f^{-1}\left(\eta_{D}^{\alpha}\right)\right](U),\left[f^{-1}\left(\eta_{D}^{\alpha}\right)\right](T)\right\}$

$$
\text { Also } \quad \begin{aligned}
{\left[f^{-1}\left(\eta_{D}^{\alpha}\right)\right](U T) } & =\eta_{D}^{\alpha}[f(U T)] \\
& =\eta_{D}^{\alpha}[f(U) f(T)] \quad(\mathrm{f} \text { is homomorphism }) \\
& \geq \min \left\{\eta_{D}^{\alpha}(f(U)), \eta_{D}^{\alpha}(f(T))\right\} \\
& =\min \left\{\left[f^{-1}\left(\eta_{D}^{\alpha}\right)\right](U),\left[f^{-1}\left(\eta_{D}^{\alpha}\right)\right](T)\right\} \\
\text { Hence, } \quad\left[f^{-1}\left(\eta_{D}^{\alpha}\right)\right](U T) & \geq \min \left\{\left[f^{-1}\left(\eta_{D}^{\alpha}\right)\right](U),\left[f^{-1}\left(\eta_{D}^{\alpha}\right)\right](T)\right\}
\end{aligned}
$$

again

$$
\begin{aligned}
{\left[f^{-1}\left(\psi_{D}^{\beta}\right)\right](U-T) } & =\psi_{D}^{\beta}[f(U-T)] \\
& =\psi_{D}^{\beta}[f(U)-f(T)] \quad(\mathrm{f} \text { is homomorphism }) \\
& \leq \max \left\{\psi_{D}^{\beta}\left(f(U), \psi_{D}^{\beta}(f(T))\right\}\right. \\
& =\max \left\{\left[f^{-1}\left(\psi_{D}^{\beta}\right)\right](U),\left[f^{-1}\left(\psi_{D}^{\beta}\right)\right](T)\right\}
\end{aligned}
$$

Hence, $\quad\left[f^{-1}\left(\psi_{D}^{\beta}\right)\right](U-T) \leq \max \left\{\left[f^{-1}\left(\psi_{D}^{\beta}\right)\right](U),\left[f^{-1}\left(\psi_{D}^{\beta}\right)\right](T)\right\}$

Also 


$$
\begin{aligned}
{\left[f^{-1}\left(\psi_{D}^{\beta}\right)\right](U T) } & =\psi_{D}^{\beta}[f(U T)] \\
& =\psi_{D}^{\beta}[f(U) f(T)] \quad(\mathrm{f} \text { is homomorphism }) \\
& \leq \max \left\{\psi_{D}^{\beta}\left(f(U), \psi_{D}^{\beta}(f(T))\right\}\right. \\
& =\max \left\{\left[f^{-1}\left(\psi_{D}^{\beta}\right)\right](U),\left[f^{-1}\left(\psi_{D}^{\beta}\right)\right](T)\right\}
\end{aligned}
$$

Hence, $\quad f^{-1}\left[\left(\psi_{D}^{\beta}\right)(U T)\right] \leq \max \left\{f^{-1}\left(\psi_{D}^{\beta}\right)(U), f^{-1}\left(\psi_{D}^{\beta}\right)(T)\right\}$

Therefore, $\mathrm{f}^{-1}(\mathrm{D})$ is a intuitionistic fuzzy HX subring of $\vartheta_{1}$.

\subsection{Theorem}

Let $\mathrm{R}_{1}$ and $\mathrm{R}_{2}$ be any two rings. Let $\mathrm{A}=\left\{\left(\mathrm{x}, \mu_{\mathrm{A}}(\mathrm{x}), \gamma_{\mathrm{A}}(\mathrm{x})\right) / \mathrm{x} \in \mathrm{R}_{1}\right\}$ and $\mathrm{B}=\left\{\left(\mathrm{y}, \alpha_{\mathrm{B}}(\mathrm{y}), \beta_{\mathrm{B}}(\mathrm{y})\right) / \mathrm{y} \in \mathrm{R}_{2}\right\}$ be any two intuitionistic fuzzy sets on $\mathrm{R}_{1}$ and $\mathrm{R}_{2}$ respectively.

Let $\mathrm{C}=\left\{\left(\mathrm{U}, \lambda_{C}^{\mu}(U), \theta_{C}^{\gamma}(U)\right) / \mathrm{U} \in \vartheta_{1}\right\}$ and $\mathrm{D}=\left\{\left(\mathrm{V}, \eta_{D}^{\alpha}(V), \psi_{D}^{\beta}(V)\right) / \mathrm{V} \in \vartheta_{2}\right\}$ be any two intuitionistic fuzzy sets in $\vartheta_{1}$ and $\vartheta_{2}$ resp. Let $f$ be an onto anti-homomorphism from $\vartheta_{1}$ to $\vartheta_{2}$. If $C$ be the intuitionistic fuzzy HX subring of $\vartheta_{1}$ then $\mathrm{f}(\mathrm{C})$ is a intuitionistic fuzzy HX subring of $\vartheta_{2}$.

Proof.

Let $\mathrm{C}$ be the intuitionistic fuzzy HX subring of $\vartheta_{1}$ then

$$
\begin{array}{cl}
\text { (i) } & \lambda_{C}^{\mu}(U-T) \geq \min \left\{\lambda_{C}^{\mu}(U), \lambda_{C}^{\mu}(T)\right\} \\
\text { (ii) } & \lambda_{C}^{\mu}(U T) \geq \min \left\{\lambda_{C}^{\mu}(U), \lambda_{C}^{\mu}(T)\right\} \\
\text { (iii) } & \theta_{C}^{\gamma}(U-T) \leq \max \left\{\lambda_{C}^{\mu}(U), \lambda_{C}^{\mu}(T)\right\} \\
\text { (iv) } & \theta_{C}^{\gamma}(U T) \leq \max \left\{\lambda_{C}^{\mu}(U), \lambda_{C}^{\mu}(T)\right\}
\end{array}
$$

To Prove that $\mathrm{f}(\mathrm{C})$ is an intuitionistic fuzzy HX subring of $\vartheta_{2}$.

$$
\begin{aligned}
& \text { Let } \mathrm{V}=\mathrm{f}(\mathrm{U}), \mathrm{W}=\mathrm{f}(\mathrm{T}) \in \vartheta_{2}, \text { where } \mathrm{U}, \mathrm{T} \in \vartheta_{1} . \\
& \begin{aligned}
& \text { Now } \eta_{D}^{\alpha}[f(U)-f(T)]= \eta_{D}^{\alpha}[f(T-U)] \quad(\mathrm{f} \text { is an anti homomorphism }) \\
&= \lambda_{C}^{\mu}[T-U] \quad(\mathrm{f} \text { is onto }) \\
& \geq \min \left\{\lambda_{C}^{\mu}(T), \lambda_{C}^{\mu}(U)\right\} \\
& \geq \min \left\{\lambda_{C}^{\mu}(U), \lambda_{C}^{\mu}(T)\right\} \\
& \geq \min \left\{\eta_{D}^{\alpha}(f(U)), \eta_{D}^{\alpha}(f(T))\right\}
\end{aligned}
\end{aligned}
$$

Hence, $\quad \eta_{D}^{\alpha}[f(U)-f(T)] \geq \min \left\{\eta_{D}^{\alpha}(f(U)), \eta_{D}^{\alpha}(f(T))\right\}$

$$
\begin{aligned}
\text { Again } \eta_{D}^{\alpha}[f(U) f(T)]= & \eta_{D}^{\alpha}[f(T U)] \quad(\mathrm{f} \text { is an anti homomorphism }) \\
= & \lambda_{C}^{\mu}[T U] \quad(\mathrm{f} \text { is onto }) \\
\geq & \min \left\{\lambda_{C}^{\mu}(T), \lambda_{C}^{\mu}(U)\right\} \\
\geq \min \left\{\lambda_{C}^{\mu}(U), \lambda_{C}^{\mu}(T)\right\} & \\
& \geq \min \left\{\eta_{D}^{\alpha}(f(U)), \eta_{D}^{\alpha}(f(T))\right\}
\end{aligned}
$$

Hence, $\quad \eta_{D}^{\alpha}[f(U) f(T)] \geq \min \left\{\eta_{D}^{\alpha}(f(U)), \eta_{D}^{\alpha}(f(T))\right\}$

Now

$$
\begin{aligned}
\psi_{D}^{\beta}[f(U)-f(T)] & =\psi_{D}^{\beta}[f(T-U)] \quad(\mathrm{f} \text { is an anti homomorphism) } \\
& =\theta_{C}^{\gamma}[T-U] \quad(\mathrm{f} \text { is onto }) \\
& \leq \max \left\{\theta_{C}^{\gamma}(T), \theta_{C}^{\gamma}(U)\right\} \\
& \leq \max \left\{\theta_{C}^{\gamma}(U), \theta_{C}^{\gamma}(T)\right\} \\
& =\max \left\{\psi_{D}^{\beta}(f(U)), \psi_{D}^{\beta}(f(T))\right\}
\end{aligned}
$$

Hence, $\quad \psi_{D}^{\beta}[f(U)-f(T)] \leq \max \left\{\psi_{D}^{\beta}(f(U)), \psi_{D}^{\beta}(f(T))\right\}$

Also

$$
\begin{aligned}
\psi_{D}^{\beta}[f(U) f(T)] & =\psi_{D}^{\beta}[f(U T)] \quad(\mathrm{f} \text { is an anti homomorphism }) \\
& =\theta_{C}^{\gamma}(U T) \quad(\mathrm{f} \text { is onto }) \\
& \leq \max \left\{\theta_{C}^{\gamma}(U), \theta_{C}^{\gamma}(T)\right\}
\end{aligned}
$$


$\begin{aligned} & =\max \left\{\psi_{D}^{\beta}(f(U)), \psi_{D}^{\beta}(f(T))\right\} \\ \text { Hence, } \quad \psi_{D}^{\beta}[f(U T)] & \leq \max \left\{\psi_{D}^{\beta}(f(U)), \psi_{D}^{\beta}(f(T))\right\}\end{aligned}$

Thus $\mathrm{D}=\mathrm{f}(\mathrm{C})$ is an intuitionistic fuzzy HX subring of $\vartheta_{2}$.

\subsection{Theorem}

Let $R_{1}$ and $R_{2}$ be any two rings. Let $A=\left\{\left(x, \mu_{A}(x), \gamma_{A}(x)\right) / x \in R_{1}\right\}$ and

$B=\left\{\left(y, \alpha_{B}(y), \beta_{B}(y)\right) / y \in R_{2}\right\}$ be any two intuitionistic fuzzy sets on $R_{1}$ and $R_{2}$ respectively.

Let $\mathrm{C}=\left\{\left(\mathrm{U}, \lambda_{C}^{\mu}(U), \theta_{C}^{\gamma}(U)\right) / \mathrm{U} \in \vartheta_{1}\right\}$ and $\mathrm{D}=\left\{\left(\mathrm{V}, \eta_{D}^{\alpha}(V), \psi_{D}^{\beta}(V)\right) / \mathrm{V} \in \vartheta_{2}\right\}$ be any two intuitionistic fuzzy sets in $\vartheta_{1}$ and $\vartheta_{2}$ resp. Let $\mathrm{f}$ be an onto anti- homomorphism from $\vartheta_{1}$ to $\vartheta_{2}$. If $\mathrm{D}$ be the intuitionistic fuzzy HX subring of $\vartheta_{2}$ then $\mathrm{f}^{-1}(\mathrm{D})$ is a intuitionistic fuzzy HX subring of $\vartheta_{1}$.

Proof.

Let $\mathrm{D}$ be the intuitionistic fuzzy HX subring of $\vartheta_{2}$ then

$$
\begin{array}{ll}
\text { (i) } & \eta_{D}^{\alpha}[V-W] \geq \min \left\{\eta_{D}^{\alpha}(V), \eta_{D}^{\alpha}(W)\right\} \\
\text { (ii) } & \eta_{D}^{\alpha}[V W] \geq \min \left\{\eta_{D}^{\alpha}(V), \eta_{D}^{\alpha}(W)\right\} \\
\text { (iii) } & \psi_{D}^{\beta}[V-W] \leq \max \left\{\psi_{D}^{\beta}(V), \psi_{D}^{\beta}(W)\right\} \\
\text { (iv) } & \psi_{D}^{\beta}[V W] \leq \max \left\{\psi_{D}^{\beta}(V), \psi_{D}^{\beta}(W)\right\}
\end{array}
$$

To Prove that $\mathrm{f}^{-1}(\mathrm{D})$ is a intuitionistic fuzzy HX subring of $\vartheta_{1}$.

Let $\mathrm{U}, \mathrm{T} \in \vartheta_{1}$ and $\mathrm{f}(\mathrm{U})=\mathrm{V}, \mathrm{f}(\mathrm{T})=\mathrm{W} \in \vartheta_{2}$.

$$
\text { Now, } \quad \begin{aligned}
{\left[f^{-1}\left(\eta_{D}^{\alpha}\right)\right](U-T) } & =\eta_{D}^{\alpha}[f(U-T)] \quad(\mathrm{f} \text { is an anti homomorphism }) \\
& =\eta_{D}^{\alpha}[f(T)-f(U)] \\
& \geq \min \left\{\eta_{D}^{\alpha}(f(T)), \eta_{D}^{\alpha}(f(U))\right\} \\
& \geq \min \left\{\eta_{D}^{\alpha}(f(U)), \eta_{D}^{\alpha}(f(T))\right\} \\
& =\min \left\{\left[f^{-1}\left(\eta_{D}^{\alpha}\right)\right](U),\left[f^{-1}\left(\eta_{D}^{\alpha}\right)\right](T)\right\}
\end{aligned}
$$

Hence, $\quad\left[f^{-1}\left(\eta_{D}^{\alpha}\right)\right](U-T) \geq \min \left\{\left[f^{-1}\left(\eta_{D}^{\alpha}\right)\right](U),\left[f^{-1}\left(\eta_{D}^{\alpha}\right)\right](T)\right\}$

Also $\quad\left[f^{-1}\left(\eta_{D}^{\alpha}\right)\right](U T)=\eta_{D}^{\alpha}[f(U T)]$

$$
\begin{aligned}
& =\eta_{D}^{\alpha}[f(T) f(U)] \quad(\mathrm{f} \text { is an anti homomorphism }) \\
& \geq \min \left\{\eta_{D}^{\alpha}(f(T)), \eta_{D}^{\alpha}(f(U))\right\} \\
& \geq \min \left\{\eta_{D}^{\alpha}(f(U)), \eta_{D}^{\alpha}(f(T))\right\} \\
& =\min \left\{\left[f^{-1}\left(\eta_{D}^{\alpha}\right)\right](U),\left[f^{-1}\left(\eta_{D}^{\alpha}\right)\right](T)\right\}
\end{aligned}
$$

Hence, $\quad\left[f^{-1}\left(\eta_{D}^{\alpha}\right)\right](U T) \geq \min \left\{\left[f^{-1}\left(\eta_{D}^{\alpha}\right)\right](U),\left[f^{-1}\left(\eta_{D}^{\alpha}\right)\right](T)\right\}$

again

Hence, $\quad\left[f^{-1}\left(\psi_{D}^{\beta}\right)\right](U-T) \leq \max \left\{\left[f^{-1}\left(\psi_{D}^{\beta}\right)\right](U),\left[f^{-1}\left(\psi_{D}^{\beta}\right)\right](T)\right\}$

Also

$$
\begin{aligned}
{\left[f^{-1}\left(\psi_{D}^{\beta}\right)\right](U T) } & =\psi_{D}^{\beta}[f(U T)] \\
& =\psi_{D}^{\beta}[f(T) f(U)] \quad(\mathrm{f} \text { is an anti homomorphism }) \\
& \leq \max \left\{\psi_{D}^{\beta}\left(f(T), \psi_{D}^{\beta}(f(U))\right\}\right. \\
& \leq \max \left\{\psi_{D}^{\beta}\left(f(U), \psi_{D}^{\beta}(f(T))\right\}\right. \\
& =\max \left\{\left[f^{-1}\left(\psi_{D}^{\beta}\right)\right](U),\left[f^{-1}\left(\psi_{D}^{\beta}\right)\right](T)\right\}
\end{aligned}
$$


Hence, $\quad f^{-1}\left[\left(\psi_{D}^{\beta}\right)(U T)\right] \leq \max \left\{f^{-1}\left(\psi_{D}^{\beta}\right)(U), f^{-1}\left(\psi_{D}^{\beta}\right)(T)\right\}$

Therefore, $f^{-1}(D)$ is a intuitionistic fuzzy HX subring of $\vartheta_{1}$.

\section{Conclusion}

In this paper we introduce the concept of intuitionistic fuzzy HX ring and discuss the basic results on intuitionistic HX subring. Further investigation may be in intuitionistic fuzzy HX ideals on HX ring. which will give a new horizon in the further study.

\section{Acknowledgements}

The authors would like to express their sincere thanks to all our friends for their help to make this paper a successful one.

\section{References}

[1]. Atanassov.K.T, intuitionistic fuzzy sets,Fuzzy Sets and System 20 (1986) ,pp 87-96.

[2]. Bing-xueYao and Yubin-Zhong, Upgrade of algebraic structure of ring, Fuzzy information and Engineering (2009)2:219-228

[3]. Bing-xueYao and Yubin-Zhong, The construction of power ring, Fuzzy information and Engineering (ICFIE),ASC 40,pp.181187,2007

[4]. Dheena.P and Mohanraaj.G, On intuitionistic fuzzy $\mathrm{k}$ ideals of semi rings, International Journal of computational cognition, Volume 9, No.2, 45-50, June 2011

[5]. Li Hong Xing, HX group, BUSEFAL, 33(4), 31-37, October 1987

[6]. $\quad$ Li Hong Xing, HX ring, BUSEFAL, 34(1) 3-8, January 1988

[7]. Liu. W.J., Fuzzy invariant subgroups and fuzzy ideals, Fuzzy sets and systems,8:133-139.

[8]. Muthuraj.R, Manikandan K.H., Sithar Selvam.P.M., Intuitionistic Q-fuzzy normal HX group ,International Journal of Mathematical Archive, Vol.2,no10.,pp 1828-1832.

[9]. Muthuraj.R, Manikandan K.H., Intuitionistic Q-fuzzy HX subgroup and $<\alpha, \beta>$ level sub HX groups, CIIT International DOI FS 122011010.

[10]. Muthuraj.R, Ramila Gandhi.N ., Induced fuzzy k-ideal and its level k-ideals on a HX ring ,IOSR Journal of Mathematics (IOSRJM) Volume 9, Issue 6 (Jan 2014), pp 138-143.

[11]. Rosenfeld. A., Fuzzy groups,J.Math.Anal.,35(1971),512-517.

[12]. Zadeh.L.A., Fuzzy sets,Information and control,8,338-353. 